\title{
The Periodontal Ligament, A Key Factor During Orthodontic Tooth Movement: Mechanical Characterization by Nanoindentation
}

\author{
Jean-Sébastien Lecomte ${ }^{1 *}$, Fabien Bornert ${ }^{2,3}$ and Delphine Wagner ${ }^{1,2,3}$ \\ ${ }^{1}$ University of Lorraine, France \\ ${ }^{2}$ Université de Strasbourg, France \\ ${ }^{3}$ Strasbourg University Hospital, France
}

*Corresponding author: Jean-Sébastien Lecomte, (LEM3), University of Lorraine, France

To Cite This Article: Jean-Sébastien Lecomte, Fabien Bornert, Delphine Wagner. The Periodontal Ligament, A Key Factor During Orthodontic Tooth Movement: Mechanical Characterization by Nanoindentation. Am J Biomed Sci \& Res. 2021 - 13(3). AJBSR.MS.ID.001880. DOI: 10.34297/AJBSR.2021.13.001880.

Received: 海 April 28, 2021; Published: 㭗 July 06, 2021

\begin{abstract}
This study concerns the determination of some mechanical properties of the periodontal ligament (PDL) by nanoindentation tests. The PDL is an essential promoter of the transmission of stresses applied to a tooth, then of bone remodeling. Few data available in the literature contradict each other. Some mechanical properties of the periodontal ligament were determined by nanoindentation and compared to the existing literature.
\end{abstract}

Keywords: Periodontal ligament; Mechanobiological phenomena; Modulus of elasticity; Nanoindentation; Orthodontic tooth movement; Bone remodeling; Finite element analysis

Abbreviations: PDL: Periodontal Ligament; OPS: Oxide Polishing Suspension

\section{Introduction}

Dental displacement is primarily a phenomenon linked to the periodontal ligament (PDL). This connective tissue vascularized and innervated, on an average thickness of 200 microns, surrounds the teeth and connects them to the surrounding bone, (Figure 1) [1]. The mechanical efforts applied on teeth will induce volume changes within the PDL, with the major consequence of altering blood flow. Depending on the deformation of the PDL, either by compression or tension, bone resorption or apposition, respectively will be activated. This combination of phenomena allows the promotion of tooth movement by orthodontic appliances. Orthodontists would like to know the effect of the forces they apply on the PDL to try to apply ideal forces and thus optimize their treatments [2,3]. Since it is difficult or impossible to quantify these phenomena in vivo, numerical models based on the finite element analysis offer important data to help practitioners [4]. However, it is necessary to identify the mechanical behavior of the involved structures as teeth, bone, and PDL. The scientific lock is in the identification of the mechanical properties including the Young's modulus, not of the teeth or the bone, but of the PDL. The literature reports a wide range from 0.07MPa to $1750 \mathrm{MPa}$ [5]. This disparity can be explained by the complexity of the PDL, its small size, its nonlinear behavior and the heterogeneity of the methods used [6]. Conventional tests such as uniaxial traction or compression are unable to capture these microscopic heterogeneities. Nanoindentation responds to these limitations and offers new perspectives for the mechanical characterization of soft tissues [7-10]. However, protocols lack consensus. The objective of our study is to quantify the Young's modulus of PDL from porcine samples by nanoindentation. 


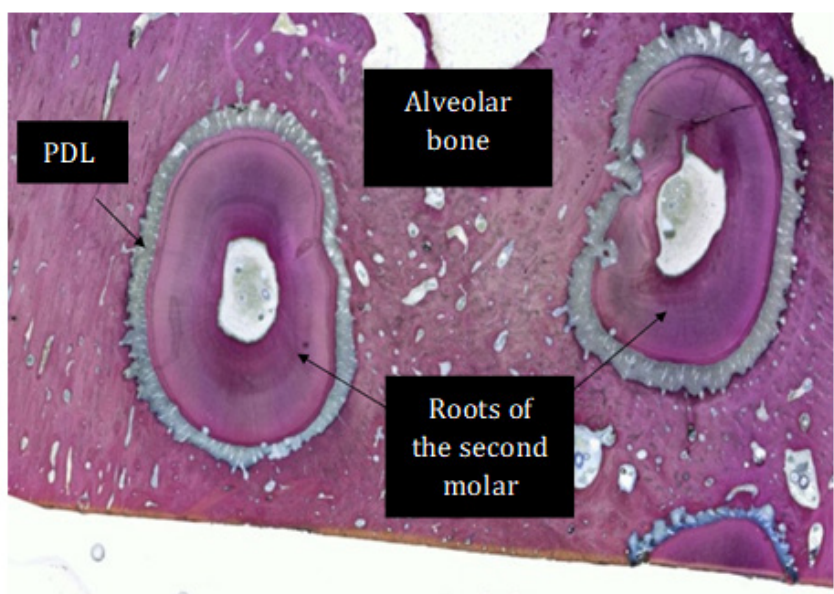

$500 \stackrel{\mapsto m}{\mu m}$

Figure 1: Histological cross section through the right maxillary second molar of a porcine sample.

\section{Material and Methods}

Porcine second molars were used and frozen at $-20{ }^{\circ} \mathrm{C}$ to facilitate their cutting without tissue damage and then sliced by ultramicrotomy (EM UC7, Leica, Germany). Samples were polished using sanding paper of decreasing granulometry and cleaned ultrasonically with distilled water between each polishing step. the conservation medium was a $10 \%$ neutral buffered formalin solution, then for the experiment they were immersed in a $5 \%$ saline solution. Nanoindentation were performed on Anton-paar's $\mathrm{NHT}^{2}$ indenter (Anton Paar TriTec NHT2, Anton Paar, Peseux, Switzerland) with a berkovich diamond indenter. The indentations were carried out with maximum load of $1 \mathrm{mN}$ and loading/ unloading rate $3 \mathrm{mN} / \mathrm{min}$ [11-12].

\section{Results and Discussion}

Load and displacement are monitored during the loading and unloading (Figure 2), and properties such as hardness and reduced modulus are calculated. The stiffness at peak load $\left(S=\frac{d P}{d h}\right)_{h_{\max }}$ is calculated as the slope of the unloading curve. The projected area $\left(A_{p}\right)$ is obtained via a calibration function (here $A_{p}=\left(24.5 h_{c}^{2}+818.34 h_{c}+9957 h_{c}^{1 / 2}\right)$ with $h_{c}$ the contact penetration depth calculated as: $h_{c}=h_{\max }-\frac{3}{4} \frac{P_{\max }}{S}$ These two parameters are used to compute the reduced modulus, according to formulas cited in Figure 2.

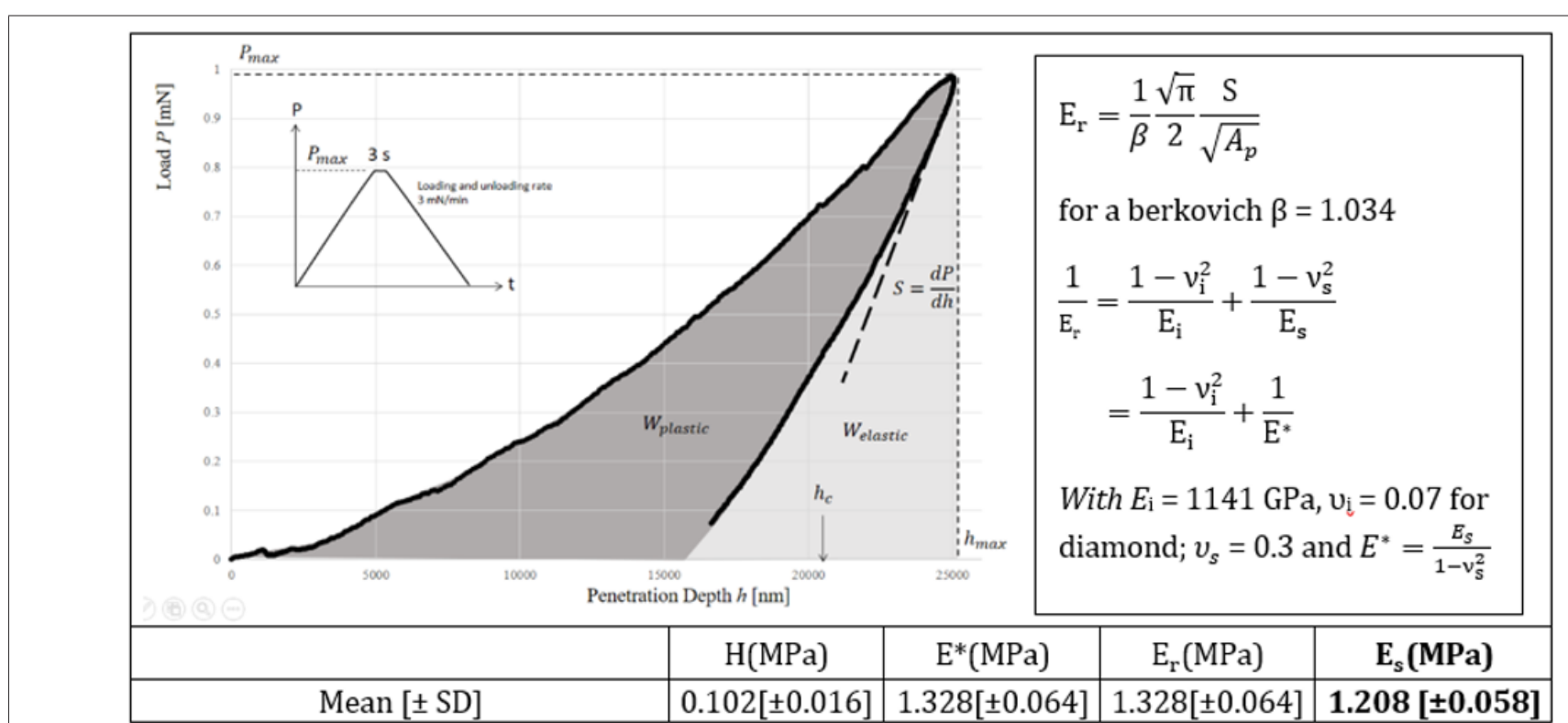

Figure 2: Load-Penetration depth curve of PDL $\left(\mathrm{W}_{\text {elastic }}\right.$ and $\mathrm{W}_{\text {plastic }}$ are the elastic and plastic part of indentation work respectively

S: Stiffness; $h_{\max }$ and $h_{c}$ : Maxi and Contact Depth Penetration Respectively; H: Hardness in MPa; $E^{*}$ : Reduced Modulus; $E_{r}$ : Plane Strain Modulus; $\mathrm{E}_{\mathrm{s}}$ : Young Modulus; u: Poisson's ratio; SD: Standard Deviation. 
The data from further human experimentations would allow the implementation of numerical models, to reproduce more accurately the clinical situations, in particular the bone remodeling contributing to dental movements.

\section{Conclusion}

The PDL is the key element in the transmission of mechanical forces caused by orthodontic appliances and inducing bone remodeling. Its mechanical characterization is subject to important limitations but nanoindentation offers new opportunities for experimental studies. From our results, Young's modulus of porcine PDL has been quantified at $1.208 \mathrm{MPa}[ \pm 0.058]$ on average.

\section{References}

1. Pietrzak G, Curnier A, Botsis J, Scherrer S, Wiskott A, et al. (2002) A nonlinear elastic model of the periodontal ligament and its numerical calibration for the study of tooth mobility. Comput Methods Biomech Biomed Engin 5(2): 91-100.

2. Natali AN, Carniel EL, Pavan PG, Sander FG, Dorow C, et al. (2008) A visco-hyperelastic-damage constitutive model for the analysis of the biomechanical response of the periodontal ligament. J Biomech Eng 130(3): 031004.

3. Slomka N, Vardimon AD, Gefen A, Pilo R, Bourauel C, et al. (2008) Time-related PDL: viscoelastic response during initial orthodontic tooth movement of a tooth with functioning interproximal contact-a mathematical model. J Biomech 41(9):1871-1877.
4. Huang H, Tang W, Yan B, Wu B, Cao D (2016) Mechanical responses of the periodontal ligament based on an exponential hyperelastic model: a combined experimental and finite element method. Comput Methods Biomech Biomed Engin 19(2):188-198.

5. Minch L (2013) Material properties of periodontal ligaments. Postepy Hig Med Dosw 11(67): 1261-1264.

6. Rees JS, Jacobsen PH (1997) Elastic modulus of the periodontal ligament. Biomaterials 18(14): 995-999.

7. Huang H, Tang W, Tan Q, Yan B (2017) Development and parameter identification of a visco-hyperelastic model for the periodontal ligament. J Mech Behav Biomed Mater 68: 210-215.

8. Oyen M, Taylor D (2009) Special issue on nanoindentation of biological materials. J Mech Behav Biomed Mater 2(4): 311.

9. Qian L, Zhao H (2018) Nanoindentation of soft biological materials. Micromachines (Basel) 9(12): 654.

10. Ashrafi H, Shariyat M (2016) A nano-indentation identification technique for viscoelastic constitutive characteristics of periodontal ligaments. J Biomed Phys Eng 6(2): 109-118.

11. Oliver WC, Pharr GM (1992) An improved technique for determining hardness and elastic modulus using load and displacement sensing indentation experiments. J Mater Res 7: 1564-1583.

12. Ebenstein DM, Pruitt LA (2004) Nanoindentation of soft hydrated materials for application to vascular tissues. J Biomed Mater Res A 69(2): 222-232. 